\title{
Strut-induced flow separation in an endostent
}

\author{
Gary Dobson, MD · Clifton R. Johnston, PhD • \\ Andrew Walker, MGIS
}

Received: 28 November 2008/Revised: 11 December 2008/Accepted: 11 December 2008/Published online: 28 January 2009

(C) Canadian Anesthesiologists' Society 2009

\section{Introduction}

Based on theoretical modelling and in vitro experiments, we can expect hemodynamic perturbations secondary to placement of intravascular stents. ${ }^{1}$ The development of flow separation along the stent wall is among the potential changes secondary to the presence of struts.

\section{Case}

A 64-year-old woman underwent bridge stenting of her thoracic aorta for management of a type 3 endoleak. Following deployment of the stent, successful exclusion of her thoracic aortic aneurysm was confirmed using echocontrast aortography. The presence of strut-induced flow separation was seen in the middle of the stent during diastole (video, available as Additional Material at www.springer.com/ 12630) (Fig. 1). Off-line analysis of the sequential frames with echo particle image velocimetry (ePIV) confirmed the presence of flow recirculation at the downstream strut and identified a proximally located secondary disturbance (Fig. 2).

Electronic supplementary material The online version of this article (doi:10.1007/s12630-009-9043-8) contains supplementary material, which is available to authorized users.

G. Dobson, MD $(\varangle)$ · C. R. Johnston, PhD · A. Walker, MGIS

University of Calgary, Calgary, Canada

e-mail: gary.dobson@albertahealthservices.ca

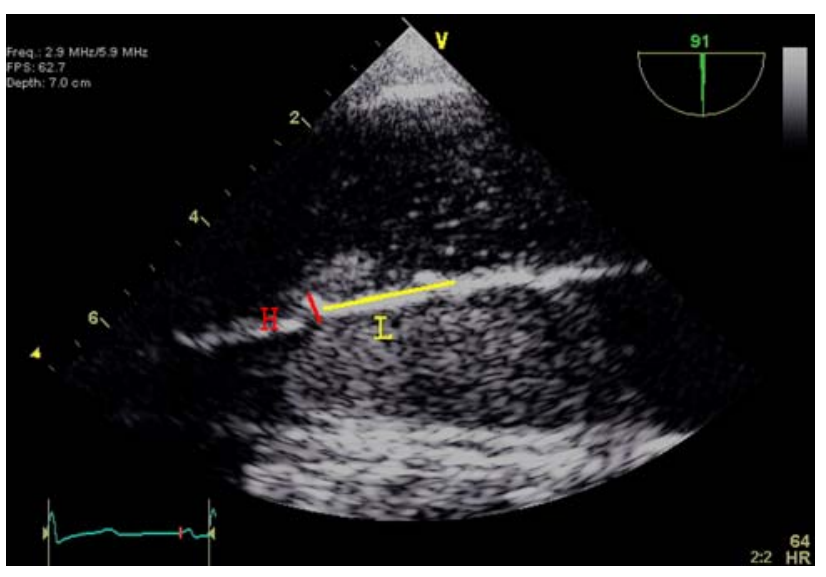

Fig. 1 Still image of strut-induced flow recirculation. The height $(\mathrm{H})$ of the strut is $0.4 \mathrm{~cm}$ and the inter-strut length (L) is $1.7 \mathrm{~cm}$

\section{Discussion}

Endovascular stenting has become the standard of care in the management of many arterial diseases. Characterization of the hemodynamic changes induced through the insertion of arterial stents has been slow. The development of strut-induced flow separation has been predicted but has not previously been demonstrated in humans. The presence of two vortices is expected when the ratio of stent height to inter-stent length is greater than three, ${ }^{2}$ as is the case here. While flow separation may be of little clinical consequence in large calibre covered stents, its presence in smaller vessels is believed to be responsible for the development of neointimal hyperplasia and graft failure. With further refinement, ePIV will allow for quantification of flow patterns throughout the cardiac cycle and will permit 
Fig. 2 Echo particle image velocimetry (ePIV) derived image of the thoracic aorta using two sequential echo contrast aortography frames in diastole that displays the presence of flow recirculation at the downstream strut and a second proximally located flow disturbance

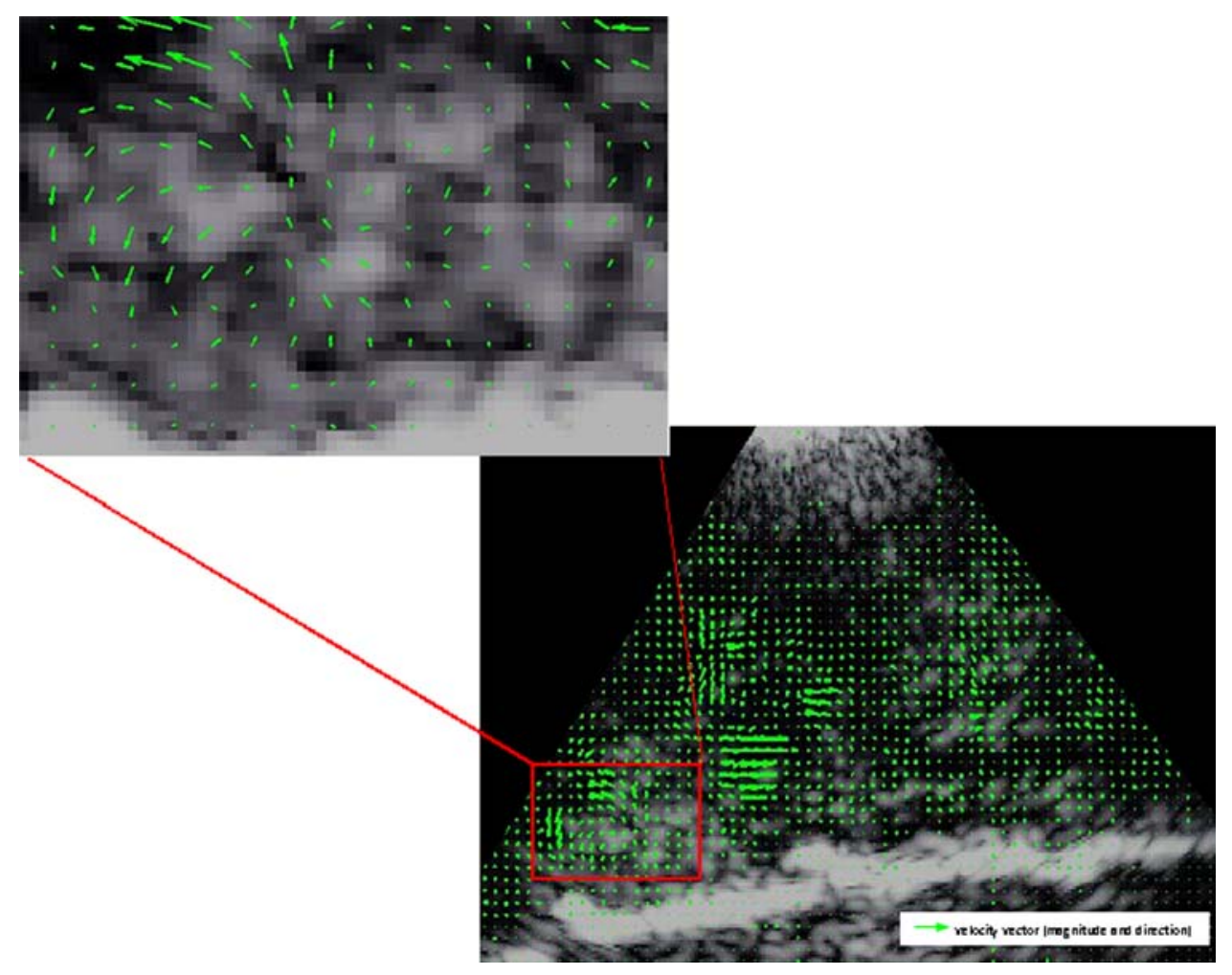

greater spatial resolution than the current $0.3 \mathrm{~mm}$. This will add to our understanding of arterial mechanics, as it has for left ventricular systolic dysfunction. ${ }^{3}$

Conflicts of interest None declared.

\section{References}

1. Moore J Jr, Berry JL. Fluid and solid mechanical implications of vascular stenting. Ann Biomed Eng 2002; 30: 498-508.
2. Berry JL, Santamarina A, Moore JE Jr, Roychowdhury S, Routh $W D$. Experimental and computational flow evaluation of coronary stents. Ann Biomed Eng 2000; 28: 386-98.

3. Hong GR, Pedrizzetti G, Tonti G, et al. Characterization and quantification of vortex flow in the human left ventricle by contrast echocardiography using vector particle image velocimetry. J Am Coll Cardiol Img 2008; 1: 705-17. 